\title{
Analysis of the colorimetric properties of goniochromatic colors using the MacAdam limits under different light sources
}

\author{
Esther Perales, ${ }^{1, *}$ Elísabet Chorro, ${ }^{1}$ Werner R. Cramer, ${ }^{2}$ \\ and Francisco M. Martínez-Verdú \\ ${ }^{1}$ Department of Optics, Pharmacology and Anatomy, University of Alicante, \\ Carretera de San Vicente del Raspeig s/n 03690, Alicante, Spain \\ ${ }^{2}$ Consultant for interference pigments, Hafenweg 22, D-48155 Muenster, Germany \\ ${ }^{*}$ Corresponding author: esther.perales@ua.es
}

Received 11 November 2010; revised 23 March 2011; accepted 28 May 2011; posted 4 August 2011 (Doc. ID 138062); published 15 September 2011

\begin{abstract}
Technological innovation in all areas has led to the appearance in recent years of new metallic and pearlescent materials, yet no exhaustive studies have been conducted to assess their colorimetric capabilities. The chromatic variability of these special-effect pigments may largely be due to the three-dimensional effect of their curved shapes and orientations when they are directionally or diffusely illuminated. Our study examines goniochromatic colors using the optimal colors (MacAdam limits) associated with normal colors (photometric scale of relative spectral reflectance from 0 to 1) under certain conventional illuminants and other light sources. From a database of 91 metallic and interference samples and using a multigonio-spectrophotometer, we analyzed samples with lightness values of more than 100 and others with lightness values of less than 100 , but with higher chromaticities than optimal colors, which places them beyond the MacAdam limits. Our study thus demonstrates the existence of chromatic perceptions beyond the normal solid color associated with these materials and independent of the light source. The challenge for future research, therefore, is to replicate and render these color appearances in current and future color reproduction technologies for computer graphics. () 2011 Optical Society of America OCIS codes: $\quad 160.1190,330.1710,330.1720,300.6550$.
\end{abstract}

\section{Introduction}

In recent years, technological innovation in all areas has led, among other things, to the appearance of new materials such as metallic and pearlescent objects developed from special-effect pigments that produce goniochromatic effects, i.e. they present notable color changes under different illuminationviewing conditions These pigments are used in many industrial activities, such as automotive coatings, cosmetics, plastics, security inks, building materials,

0003-6935/11/275271-08\$15.00/0

(C) 2011 Optical Society of America and the visual simulation of virtual environments. Their popularity is due to the fascinating interplay of colors and to effects produced by the various materials used in their layered structures [1-3]. Refractions and reflections of light at and within these layers cause interferences that yield certain colors [4] in an attempt to replicate natural colors seen in lesser animals such as butterflies and insects [5].

Interference pigments can be classed by either the manufacturing method used or by their structure. Substances such as titanium dioxide or iron oxide that have high indices of refraction may, for example, be deposited on a transparent substrate such as mica, as in the case of Iriodin, silicon dioxide, as 
in the case of Colorstream, or aluminum oxide, as in the case of Xirallic. Such pigments are produced using wet-chemical processes, whereas those with an aluminum layer as an internal reflector (Variochrom, ChromaFlair, and SpectraFlair) are manufactured in a high vacuum. Liquid crystals are also classed as interference pigments. Aluminum pigments are based on pure aluminum powder, which is broken down in a dry or wet milling process. Depending on the starting material, different types of patterns are obtained such as "corn flakes", which have an uneven platelet structure, or discs that are known as "silver dollars". Unlike interference pigments based on transparent substrate, these materials are not transparent.

The optical behavior of these materials is determined by the spectral bidirectional reflectance distribution function (BRDF), defined as the spectral ratio between the radiance of the sample in a given direction and the irradiance over that sample. Some instruments are designed to measure the spatial distribution of the reflectance factor in different geometrical configurations. The geometries needed for evaluating and observing interference and metallic pigments may be derived from their properties. Measurements are made at specified angular intervals, referred to as the angle of specular reflection, which yields a series of measured spectral data. Analyses of data acquired for three or four aspecular angles (the difference between the angle of specular reflection and the angle of observation) are frequently regarded as sufficient, although they cover and use a mere fraction of the colorimetric data available. As the color shift toward shorter wavelengths is a property peculiar to the interference pigment involved, it should be used in visually and instrumentally characterizing interference pigments. This means that different angles of illumination are required and measurements close to the angle of specular reflection, at an aspecular angle of $15^{\circ}$, should be made for each one. Additional measurements for a constant $45^{\circ}$ angle of illumination and various aspecular angles are needed to acquire overall color impressions. In this way, measurements made at various angles of illumination for the same difference angle $\left( \pm 15^{\circ}\right)$ with respect to the specular direction produce an interference line that is peculiar to the particular interference pigment involved. Measurements made at a constant angle of illumination (e.g. $45^{\circ}$ ) for various angles of observation and difference angles produce an aspecular line (see Fig. 1). Together, those two lines resemble an anchor, where neither is necessarily a straight line. Although aspecular lines usually all have a uniform shape, they may well extend over several quadrants of the $a * b *$-coordinate system in the case of diffraction pigments.

As can be seen in Fig. 2, a goniochromatic sample changes hue and chroma along the interference line, but not as much along the aspecular line. On the contrary, the lightness variation is usually greater along the aspecular line than along the interference line.
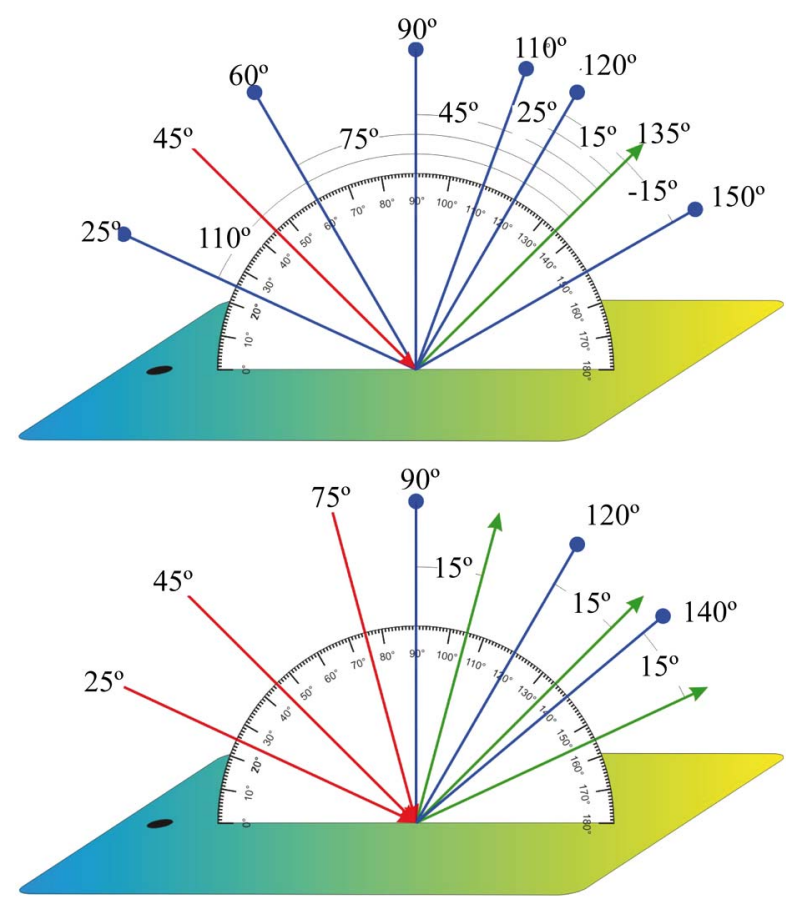

Fig. 1. (Color online) Illumination and observation angles of the measures of the Datacolor MultiFX10 gonio-spectrophotometer in accordance with the DIN 6175-2 and ASTM E2194 standards. Top: aspecular line; bottom: interference line.

For instance, in both examples relating to Fig. 2, the effect of pigment formulation can be seen. In the first case, the color formulation is pure, with only special-effect pigment, so the lightness values are higher than 100 for certain measurement geometries. In contrast, the color formulation for the second
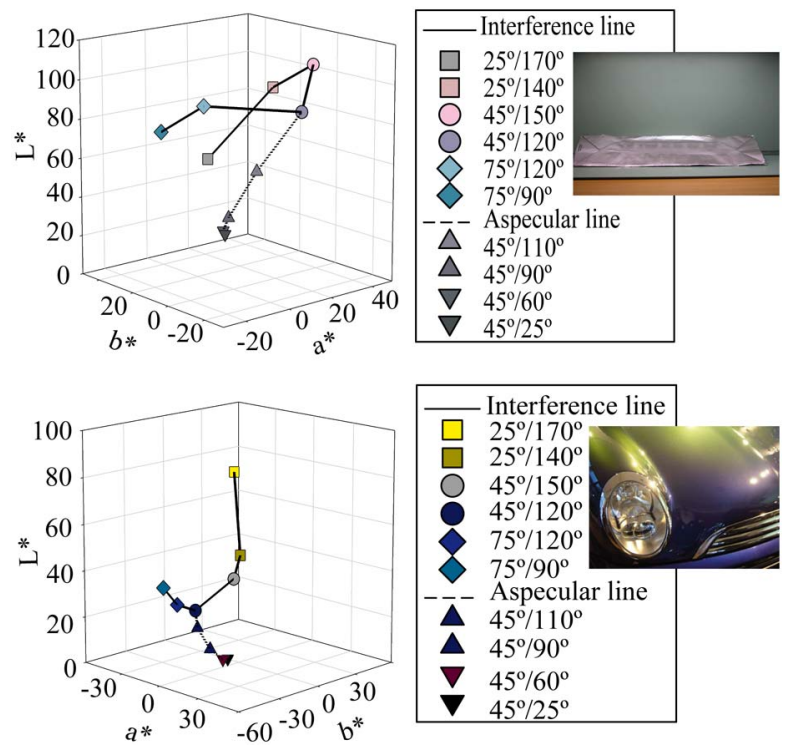

Fig. 2. (Color online) Color shifts encoded in CIELAB color space of two goniochromatic samples belong to two conventional objects showing the interference and aspecular lines obtained from a multi-gonio-spectrophotometer with 10 measurement geometries (Datacolor MultiFX10). Top: Colorstream pigment (pure formulation) belong to a piece of a handbag. Bottom: Flame Blue pigment (mixed formulation) belonging to a car body. 
case is mixed, sharing the same special-effect pigment as the first case, but incorporating certain absorbing pigments, so the lightness values are lower than 100, but have higher chroma values than those of the first case.

Although in daily situations we usually perceive complex scenes with diffuse rather than directional illumination, many conventional objects (automotive coatings, plastics, cosmetics, etc.) are manufactured with semiflat or curved shapes, even using many different orientations as regards to observer position. For instance, these optical and visual behaviors may also be slightly different by simply changing the orientation of the sample and applying the same measurement geometry, particularly for pearlescent objects [6]. Therefore, to reach a full and exhaustive understanding of the optical and perceptual behavior of these special-effect pigments and their colored materials, the complexity of crossing object shape and orientation, object material, and illumination geometry needs to be reduced.

On the other hand, according to traditional color science, the colors distinguishable by the human visual system define a three-dimensional (3D) structure called a color solid. The colors that define the limits are known as optimal colors and were studied by MacAdam [7,8]. These colors are associated with the normal reflectance/transmittance spectrum (photometric scale from 0 to $100 \%$ ), with maximum colorfulness for a given luminance factor $(Y$, lower than $100 \%$ ). The main characteristic of optimal colors is the shape of their reflectance curve. Its possible values are zero and one, with only two possible transitions between these two values throughout the visible spectrum. Two types of optimal colors can therefore be distinguished: type 1 , with a mountainlike spectral profile and type 2 , with a valleylike spectral profile. However, a recent theorem has been proved from which the number of transitions depends on the shape of the cone fundamentals [9]. However, the completed spectral contour resulting from the color matching functions adopted by the CIE as the standard colorimetric observer is convex. This indicates that, for this observer the twotransition assumption holds true. Optimal colors do not really exist; that is, they are not found in nature, or cannot be obtained by means of colorant formulation as the reflectance curve is extremely abrupt. Figure 3 shows the differences between optimal reflectances and reflectances from a real color sample. However, optimal colors delimit the conventional color solid of human perception, and evaluate the colorimetric quality of colorants [10-15]: when colorants approach the MacAdam limits, a greater range of reproducible colors can be obtained (color gamut). For instance, in 1980 M. R. Pointer [16,17] used these colorimetric data to compare several industrial color gamuts.

MacAdam worked in the CIE-xy chromaticity diagram. Since the CIE-XYZ color space is not visually uniform, it is better to calculate the color solid in a more perceptual uniform color space. CIELAB is a uniform color space that is widely used in industry as it allows the color solid to be visualized more realistically. The color solid can be obtained for any illuminant or light source.

As goniochromism is the effect of abrupt color depending on the illumination/observation angle, it is interesting to consider whether these color variations are inside or outside the Rösch-MacAdam color solid and whether this depends on the pigment type, measurement geometry, or light source used. Providing an answer to these two questions is the main objective of this work, i.e. to analyze the colorimetric characteristics of goniochromatic samples using the theoretical color solid associated with a standard observer and with different light sources associated with normal colors. This will make it possible to
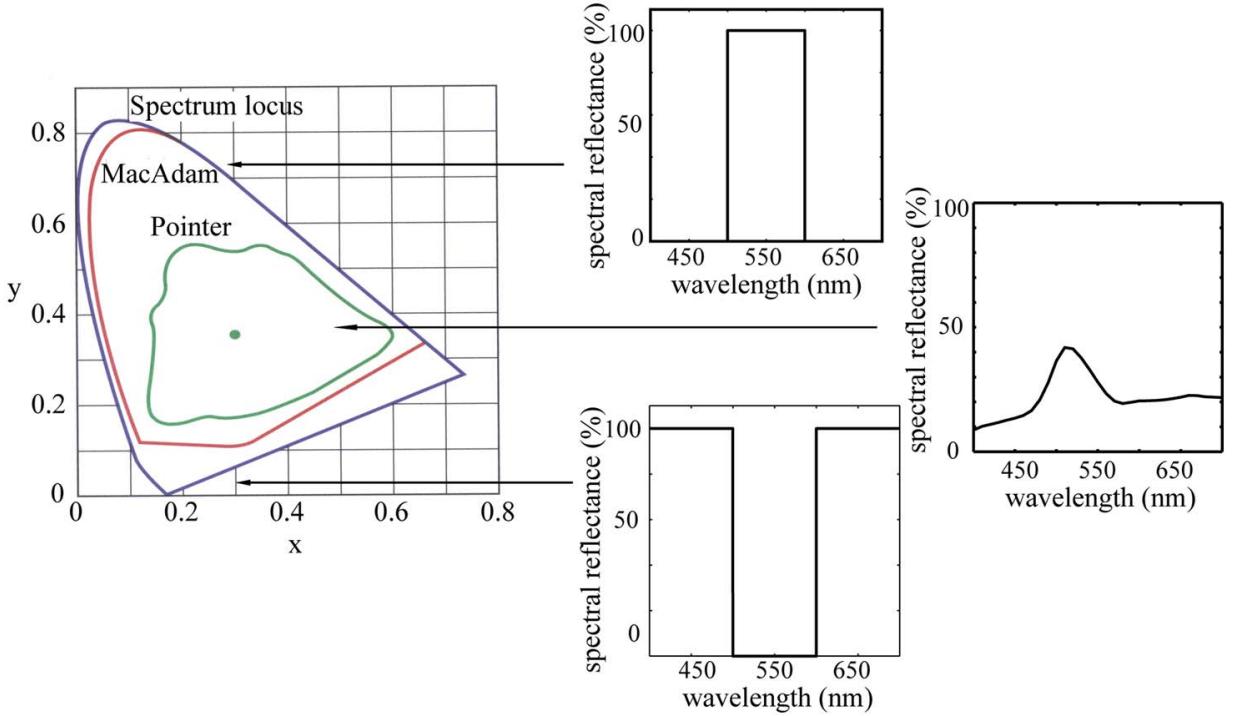

Fig. 3. (Color online) Differences between the spectral reflectance curves associated with optimal colors and a real color. 
determine the colorimetric behavior of goniochromatic samples in relation to the light source and measurement geometry.

\section{Materials and Methods}

Our goniochromatic database comprises 91 metallic and interference color samples collected from the technical color charts of various manufacturers. Each spectral reflectance relating to a standard matte and nonfluorescent white was read by the Datacolor MultiFX10 multi-gonio-spectrophotometer. This instrument has ten illumination/observation geometries in accordance with the ASTM 2194 and DIN 6175-2 standards $[18,19]$.

Based on the relative spectral reflectance curves associated with each measurement geometry, the CIELAB values were calculated by taking the CIE 1931-XYZ standard observer [20] and different light sources (D65, A, F11, HP1 and wLED) into account. The correlated color temperatures $\left(T_{c}\right)$ and CIE general color rendering indexes $\left(R_{a}\right)$ used were $6500 \mathrm{~K}$, $2856 \mathrm{~K}, 4000 \mathrm{~K}, 1959 \mathrm{~K}$, and $7800 \mathrm{~K}$, and 100,100 , 83,8 and 81, respectively. These light sources represent daylight and incandescent illumination, typical fluorescent lamps, typical high-pressure metal halide lamps, and illumination based on white LEDs that are in great demand on the market. How the correlated color temperature and color quality (color rendering) of the used light sources interacted with the goniochromatic effects in our database was also studied.

The theoretical color solid obtained from the optimal colors was taken into account when evaluating the colorimetric characteristics of the goniochromatic samples. Therefore, the color solid associated with the CIE 1931 standard observer and different illuminants, was calculated $[21,22]$, plotting the 91 goniochromatic samples together with the corresponding color solids for each illuminant and measurement geometry. However, the same color solid was considered for each measurement geometry because the Rösch-MacAdam theorem is valid and applicable regardless of the type of color measurement. Furthermore, in these 3D plots ( $L *$ versus $a *$ versus $b *$ ), the goniochromatic samples were shown with the MacAdam limits in constant lightness profiles to allow for a more detailed study and to analyze the chromatic perceptions obtained with these materials.

Therefore, even though the gonio database of 91 samples provides a large amount of color data, with ten measurement geometries and five light sources, determining whether CIELAB values are inside or outside the (normal) MacAdam limits is a straightforward matter.

\section{Results}

Firstly, color solids are shown together with the goniochromatic samples in the CIELAB color space under the illuminant D65 for all measurement geometries. As shown in Fig. 4, there are samples with lightness values greater than 100 , around $L *=135$, for the $45^{\circ} / 150^{\circ}$ and $75^{\circ} / 120^{\circ}$ measurement geometries, which are associated with the interference line. With the exception of the $25^{\circ} / 170^{\circ}$ measurement geometry (see Fig. 5), which is a very flat geometry of the interference line $\left( \pm 15^{\circ}\right)$, the other geometries of this line show gonio samples beyond the MacAdam limits due to the existence of additive color mixing near the specular direction up to $20^{\circ}-25^{\circ}$. These selected colors are therefore very different to the conventional colors produced by subtractive color mixing, and are obtained in the same samples for the aspecular angles. The results relating to the $25^{\circ} / 170^{\circ}$ geometry (shown in Fig. 5) are not plausible due to optical laws of interference, which we consider
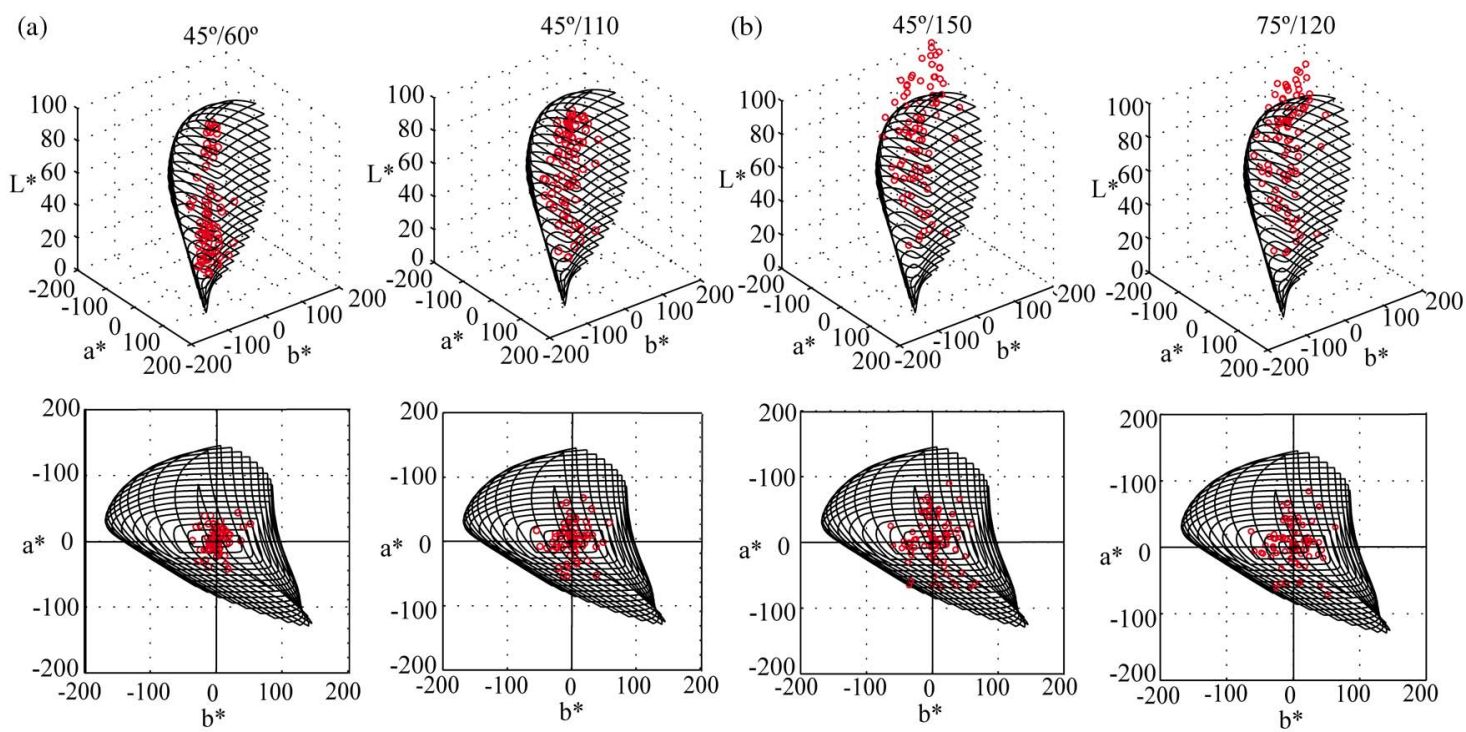

Fig. 4. (Color online) Rösch-MacAdam color solid associated with the CIE 1931 standard observer and the D65 illuminant together with the 91 goniochromatic samples measured by the Datacolor FX10 multi-gonio-spectrophotometer with different measurement geometries. a) Aspecular line. b) Interference line. 

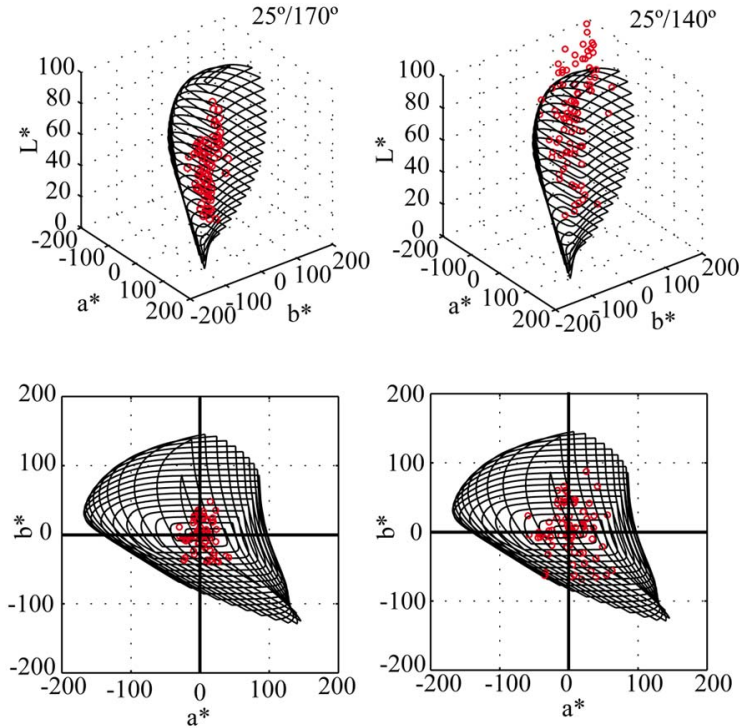

Fig. 5. (Color online) Rösch-MacAdam color solid associated with the CIE 1931 standard observer and the D65 illuminant together with the 91 goniochromatic samples measured by the Datacolor FX10 multi-gonio-spectrophotometer for the $25^{\circ} / 170^{\circ}$ and $25^{\circ} / 140^{\circ}$ measurement geometries.

to be more or less artifacts caused by the proximity of angle $180^{\circ}$ and/or by instrumental error in the angular tolerances for this measurement geometry. In contrast, and reinforcing our argument, the other measurement geometry for the same illumination direction of $25^{\circ} / 140^{\circ}$ clearly shows many gonio samples beyond MacAdam limits, as shown in Fig. $\underline{5}$. As a result, the color encodings relating to the aspecular line seem to be inside the corresponding color solid, whereas the color encodings relating to the interference line are sometimes outside the corresponding color solid.

It is important to bear in mind that in the $a *$ versus $b *$ chromatic diagram, most of the samples are less saturated than what the human visual system can distinguish, although there are some samples that are close to MacAdam limits $\left(45^{\circ} / 150^{\circ}\right.$, $75^{\circ} / 120^{\circ}$, etc.). However, to check this fact, the 91 samples must be compared with the MacAdam limits at constant lightness profiles. Figure 6 shows four constant lightness planes $(L *=30,6 \overline{0}, 90,99)$ for some of the measurement geometries, with some of the samples beyond the MacAdam limits. It seems logical to assume that it is possible to find samples outside the lightness scale, as some samples have reflectance factors of more than $100 \%$ in some spectral bands and therefore have a lightness value of more than 100 . However, it is interesting to see samples with lightness values of less than $L *<100$ that are beyond MacAdam limits. To study this behavior in more detail, Figs. $\underline{7}$ and $\underline{8}$ show the spectral reflectance of 17 samples that are beyond MacAdam limits, with $L *$ higher and lower than 100 for some measurement geometries of the interference line. As can be seen below, the existence of relative spectral reflectance values of more than $100 \%$ are necessary, but not sufficient, to lead to CIELAB values beyond MacAdam limits. The subsequent chromatic perception will depend on many parameters relating to the spectral profile, such as photometric range, contrast between the maximum and minimum values, the existence of a large number of peaks, etc. Here, for our examples, the photometric range of spectral reflectances is clearly more than $100 \%$, with some values even exceeding $200 \%$. Furthermore, for some of the gonio samples located outside MacAdam limits with $L *$ values of less than 100 , their reflectance spectrum is plotted in Fig. 8. It is interesting to observe how the photometric contrast or dynamic range can be the key feature to be encoded beyond conventional chromatic limits.

The other aim of this work is to evaluate this behavior using other illuminants (A, F11, HP1 and
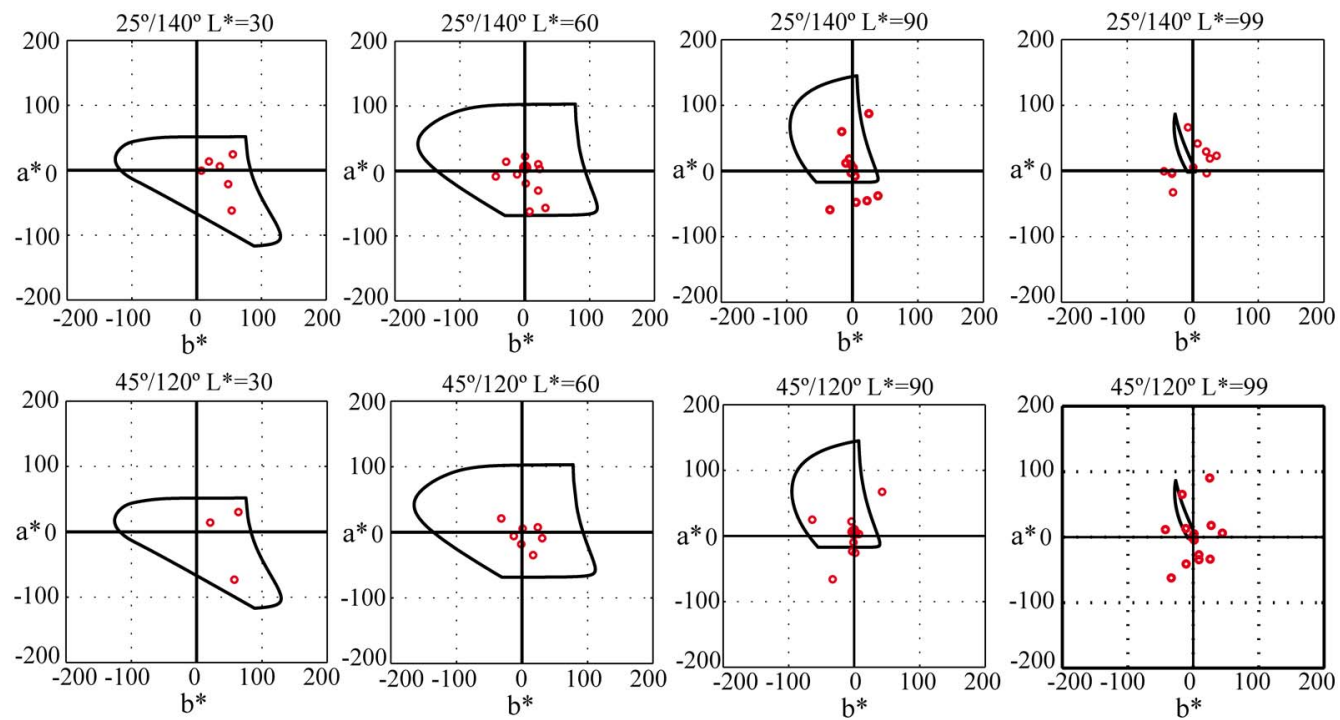

Fig. 6. (Color online) Comparison of the goniochromatic samples (red circles) with MacAdam limits (solid line) at constant lightness profiles for the measure geometries $25^{\circ} / 140^{\circ}$ and $45^{\circ} / 120^{\circ}$. 

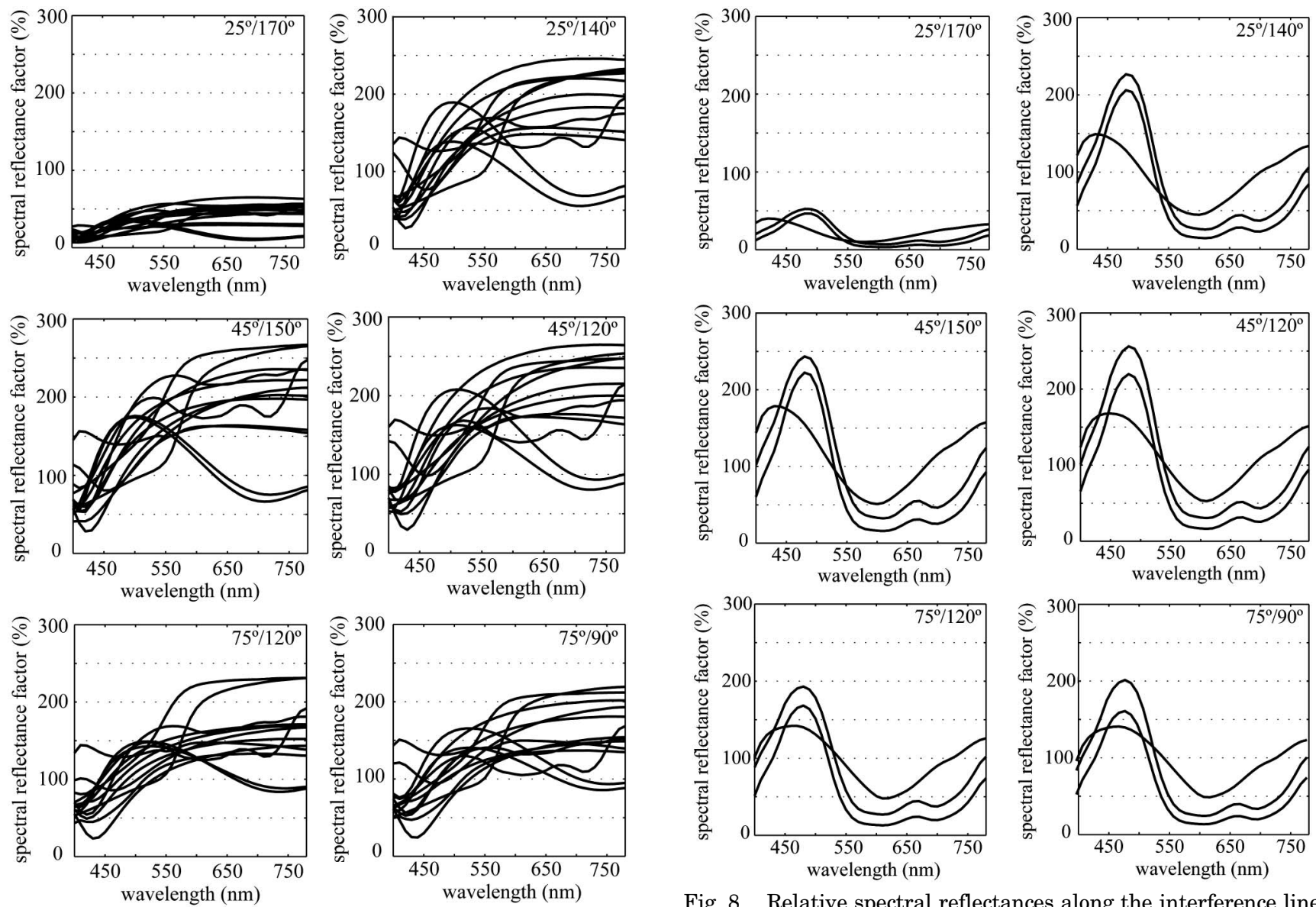

Fig. 7. Relative spectral reflectances along the interference line associated with 14 gonio samples with CIELAB values positioned outside (with $L *$ values $>100$ ) MacAdam limits.

wLED). By way of example, Fig. 9 shows the color solids relating to the goniochromatic data associated with the $45^{\circ} / 120^{\circ}$ measurement geometry, with gonio samples lying outside of MacAdam limits independently to the type and color quality of the light source. Although the spectral content of the illuminant or light source greatly affects the shape and volume of its color solid-influenced by its correlated color temperature and color rendering index, these parameters or those relating to light technology are not the main reasons for their position beyond MacAdam limits. The main reason for this significant conclusion is closely related to the physical and chemical nature of these special-effect pigments and their microarrangement inside the colored substrates (materials). However, the spectral content of these light sources (and consequently the correlated color temperature and color quality) does influence the chromatic variability of these gonio samples since the number of samples beyond MacAdam limits are not always the same, as can be observed in Fig. 10. Only three gonio samples are placed outside the color solids for the five light sources whose spectral reflectance is shown in Fig. $\underline{8}$, for the different measurement geometries associated with the interference line.
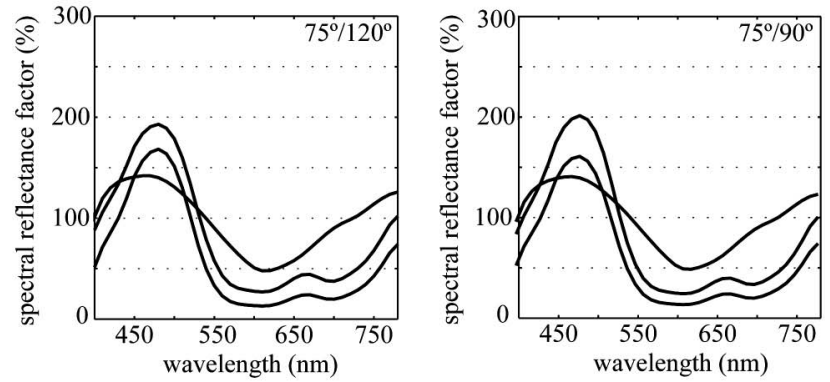

Fig. 8. Relative spectral reflectances along the interference line associated with three gonio samples with CIELAB values positioned outside (with $L *$ values $<100$, but $C_{\mathrm{ab}}^{*}>C_{\mathrm{ab}, \text { MacAdam }}^{*}$ ) MacAdam limits.

A theoretical study can then be carried out relating to spectral reflectance values, dynamic ranges, peaks, etc, to analyze how these parameters result in chromatic perception beyond the (normal) MacAdam limits. Therefore, taking this hypothesis into account, theoretical spectral reflectances located beyond the normal color solid could be predicted. The next challenge would be reproduction using current special-effect pigments which could be used to explore special-effect pigment formulations with or without additional absorbing pigments.

\section{Discussion}

In terms of conventional color science analysis, this is a highly descriptive work at the qualitative level, using conventional spectral and chromatic data and diagrams. However, at implicit levels the study is more complex as many of the calculations and color measurements combine 91 samples and 10 geometries, which are all related to the color solids associated with illuminants and real light sources. These few mathematical steps therefore make our argument a solid one for the main purpose of this work, to determine whether any chromatic perceptions lie outside the conventional chromatic MacAdam limits. As a result of this study, the answer 

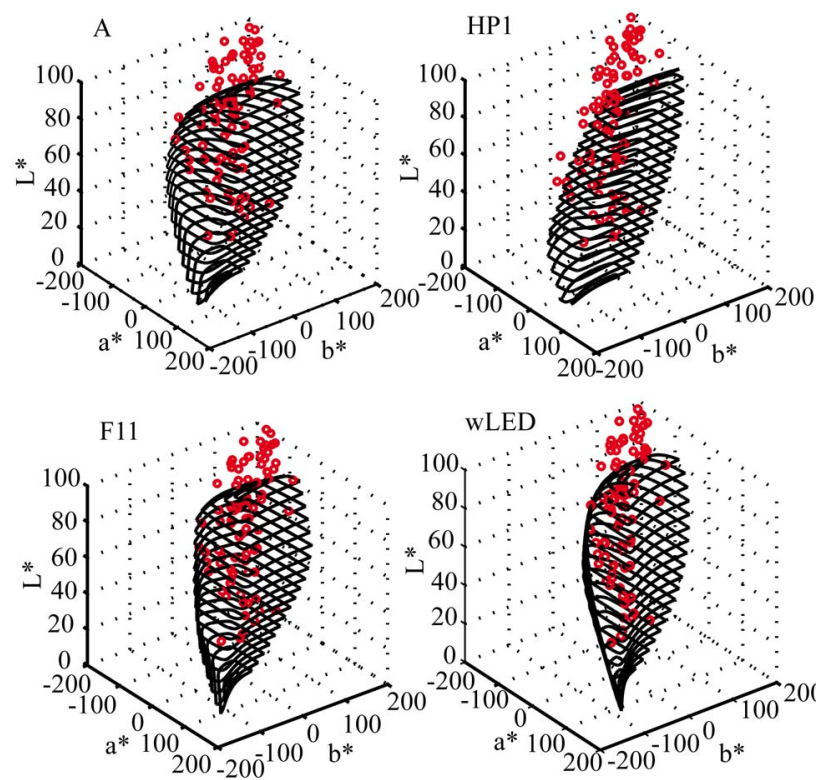

Fig. 9. (Color online) Rösch-MacAdam color solid associated with the CIE 1931 standard observer and the different light sources together with the 91 goniochromatic samples measured with Datacolor FX10 multi-gonio-spectrophotometer for the $45^{\circ} / 120^{\circ}$ measurement geometry.

to this question is clearly affirmative, although the study could be extended with a larger gonio database of special-effect pigments and a range of daily objects with these special color appearances. The
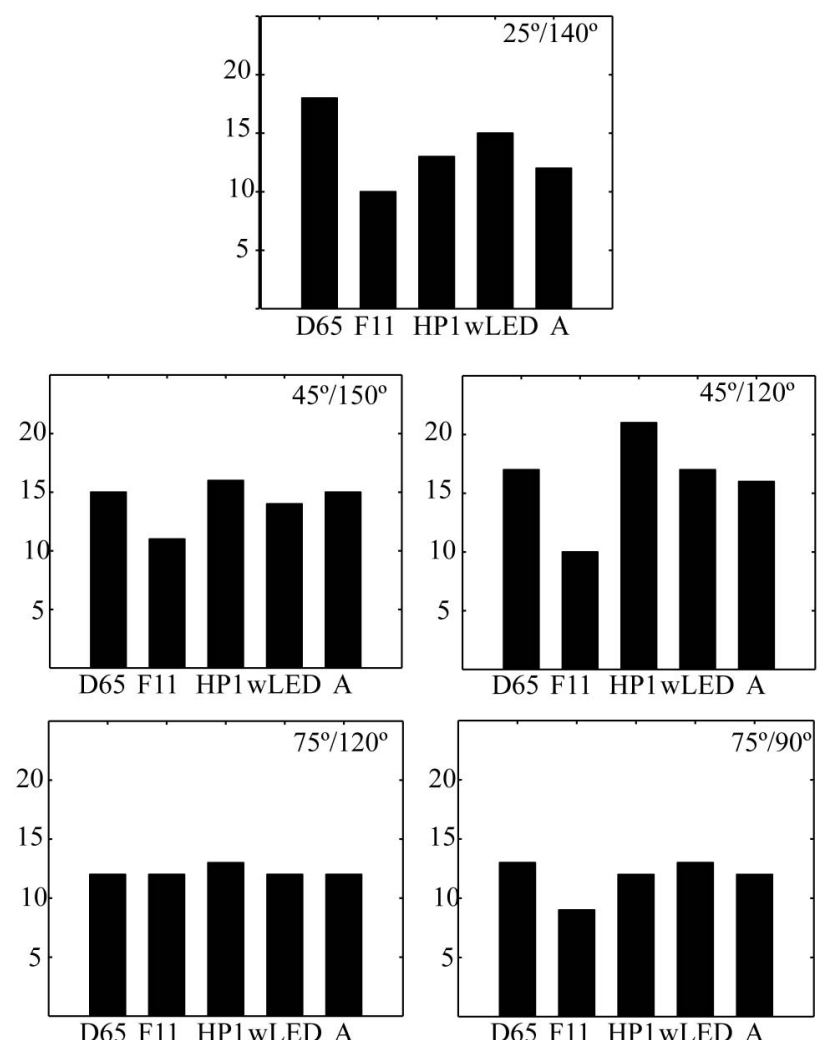

Fig. 10. Number of samples placed beyond the MacAdam limits for the different light sources and different (interference line) measurement geometries with $C_{\mathrm{ab}}^{*}>C_{\mathrm{ab}, \text { MacAdam }}^{*}$. main interest of these experiments is undoubtedly the physical and chemical nature of the special-effect pigments and their special microarrangement inside the colored materials. However, in our opinion, future works should focus on the influence of the color formulation of these special-effect pigments. In our study, we were unable to differentiate between pure and mixed color formulations that affect the outer position, and this would be an interesting direction for new research to take.

Secondly, replicating these color appearances found in real gonio samples is a major challenge for color technologies applied to computer graphics (3D cinema, virtual reality, display technologies, etc). As these special chromatic perceptions on semiflat and curved shapes of many objects are a daily occurrence, even with diffuse illumination, the question is whether these colors can be reproduced at least at a relative colorimetric level, as it seems very difficult to reproduce them at a spectral and absolute level.

Thirdly, having found that relative spectral reflectance values of more than $100 \%$ can result in chromatic perception beyond conventional limits, the next challenge would be to find an algorithm such as a pass/fail test with which to note which spectral reflectances with spectral values of more than $100 \%$ will be within or beyond MacAdam limits, taking different spectral profiles and mathematical analyses (Fourier, PCA, etc) into account. This also relates to the challenge of whether current or future specialeffect pigment technologies will be able to reproduce these spectral reflectances in formulation and what the effect would be of working with pure and mixed formulations of effect and absorbing pigments.

Finally, at an instrumental level, it would be interesting to improve coverage of the geometry range with other geometries nearer the specular angle, or even to study other interference lines, with angular values ranging from $5^{\circ}$ to $25^{\circ}$ and not only the conventional geometries of $\pm 15^{\circ}$. More information could then be obtained on how additive and subtractive color mixtures interact with effect pigments. However, all these results are associated with the incidence plane and it would be of great interest to expand this work with off-plane measurement geometries by using the X-Rite MA98 multi-goniospectrophotometer, for instance.

\section{Conclusions}

The concept of optimal color, as described in the Rösch-MacAdam theory, needs to be revised. Special-effect pigments are now used in many objects to create metallic and pearlescent appearances, in automotive and industrial paint formulations, cosmetics, plastics, and so on; their popularity is due to the fascinating interplay of colors and effects. However, until now there has been an insufficient amount of research into the colorimetric capacities of these materials that takes the chromatic variability of these special-effect pigments into account. This may largely be due to the 3D effect of their curved 
shapes and orientations in daily objects when directionally or diffusely illuminated.

This work has shown that with a large database of goniochromatic samples, a multi-goniospectrophotometer and the conventional chromatic limits for normal spectral reflectances (ranging from 0 to $100 \%$ ), known as MacAdam limits, samples exist with lightness values of less than 100 , but with chromaticities that are higher than for optimal colors and are thus beyond MacAdam limits. This conclusion is valid even for different illuminants and real light sources, in particular for measurement geometries belonging to the interference line. This result does not therefore depend on the spectral content and color quality of the illuminant and light sources, but rather on the physical and chemical nature of these special-effect pigments and their microarrangement inside the colored materials.

Consequently, this study demonstrates the existence of chromatic perceptions outside the normal color solid (MacAdam limits) associated with these materials (special-effect pigments), regardless of the type and quality of light source. The next challenge will be to replicate and render these color appearances in current and future color reproduction technologies for computer graphics. A complete theory of the spectral reflectances associated with specialeffect pigments to obtain chromatic perceptions outside the classical chromatic limits is also a future challenge. If current and future special-effect pigments could reproduce these optimal colors, it would be a great help in formulating colors.

This work is therefore useful for future research into the manufacture of colored materials with chromatic perceptions outside the classical chromatic limits. For instance, the methodology may be useful in showing potential clients for special-effect pigments the predictions of color appearance for their color designs and samples beyond the conventional color perceptibility limits.

This research; was supported by means of the grant number DPI2008-06455-C02-02 of the Spanish Ministry of Science and Innovation.

\section{References}

1. E. Kirchner, G. J. van den Kieboom, L. Njo, R. Super, and R. Gottenbos, "Observation of visual texture of metallic and pearlescent materials," Color Res. Appl. 32, 256-266 (2007).

2. G. Pfaff, Special Effect Pigments: Technical Basics And Applications (Vincentz Network, 2008), p. 218.
3. W. R. Cramer and P. Gabel, "Measuring special effects," Paint \& Coatings Industry 29, 36-46 (2001).

4. M. Douma, "Causes of Colours," retrieved March, 2010, http:// www.webexhibits.org/causesofcolor/15.html. (2008).

5. S. Berthier, Iridescences: The Physical Colors of Insects (Springer, Paris, 2006).

6. E. Chorro, E. Perales, F. M. Martinez-Verdu, J. Campos, and A. Pons, "Colorimetric and spectral evaluation of the optical anisotropy of metallic and pearlescent samples," J. Mod. Opt. 56, 1457-1465 (2009).

7. D. L. MacAdam, "Theory of the maximum visual efficiency of colored materials," J. Opt. Soc. Am. 25, 249-252 (1935).

8. D. L. MacAdam, "Maximum visual efficiency of colored materials," J. Opt. Soc. Am. 25, 361-367 (1935).

9. A. D. Logvinenko, "An object-color space," J. Vis. 9 1-23 (2009).

10. R. S. Berns, Billmeyer and Saltzman's Principles of Color Technology (Wiley, 2000).

11. R. W. G. Hunt, The Reproduction of Colour (Wiley, 2004), p. 702.

12. R. G. Kuenhi, Color Space and Its Divisions: Color Order from Antiquity to the Present (Wiley-VCH, 2003), p. 202.

13. E. Perales, E. Chorro, F. Martinez-Verdu, S. Otero, and V. de Gracia, "A new method for comparing colour gamuts among printing technologies," Imaging Sci. 56, 145-152 (2008).

14. E. Perales, E. Chorro, V. Viqueira, and F. M. Martínez-Verdú, "Estimation of the real colour gamut," in 11th Congress of the International Colour Association (AIC 2009) (Colour Society of Australia, 2009), p. 71.

15. E. Perales, F. M. Martinez-Verdu, V. Viqueira, J. FernandezReche, J. A. Diaz, and J. Uroz, "Comparison of color gamuts among several types of paper with the same printing technology," Color Res. Appl. 34, 330-332 (2009).

16. M. R. Pointer, "The gamut of real surface colors," Color Res. Appl. 5, 145-155 (1980).

17. 17.M. R. Pointer, "Request for real surface colours," Color Res. Appl. 27, 374-374 (2002).

18. ASTM-E2175-01, "Standard practice for specifying the geometry of multiangle spectrophotometers," (American Society for Testing and Materials, 2001).

19. DIN-6175-2, "Tolerances for automotive paint-Part 2: Goniochromatic paints," (Deutsches Institut für Normung, 1999).

20. CIE-15:2004, "Colorimetry," (Commision Internationale de L'Eclaraige, 2004).

21. C. Li, M. R. Luo, M. S. Cho, and J. S. Kim, "Linear programming method for computing the gamut of object color solid," J. Opt. Soc. Am. A 27, 985-991 (2010).

22. 22.F. Martinez-Verdu, E. Perales, E. Chorro, D. de Fez, V. Viqueira, and E. Gilabert, "Computation and visualization of the MacAdam limits for any lightness, hue angle, and light source," J. Opt. Soc. Am. A 24, 1501-1515 (2007). 